\title{
Gesundheit von Ärztinnen und Ärzten im Berufsalltag
}

\begin{abstract}
Physician Health in the Workplace. Physicians are exposed to a variety of risks in their everyday work. There is an obvious risk, especially in view of the current pandemic, of contracting communicable diseases like COVID-19, HIV and Hepatitis C. The commercialization of healthcare and associated cost-saving measures - particularly in the field of human resources - lead to unhealthy workloads and, correspondingly, an increased risk of suffering from psychological disorders like burnout and depression. Scientific studies reveal a correlation between psychological stress and the quality of patient care. The health of medical personnel must be given high priority in the interest of both patients and those working in the healthcare system. This requires adequate funding with staffing that is appropriate to the patient and the task at hand, thus ensuring humane and high-quality patient care. In addition, physicians must be relieved of performing non-medical tasks, and their resilience must be strengthened through individual and operational measures.
\end{abstract}

„Als Mitglied der ärztlichen Profession gelobe ich feierlich, mein Leben in den Dienst der Menschlichkeit zu stellen. Die Gesundheit und das Wohlergehen meiner Patientin oder meines Patienten werden mein oberstes Anliegen sein. "So beginnt das Genfer Gelöbnis, das 1948 auf der 2. Generalversammlung des Weltärztebundes in Genf verabschiedet wurde. Auf der ganzen Welt berufen sich Ärztinnen und Ärzte auf die Deklaration von Genf. Das Gelöbnis fasst die grundlegenden ethischen Prinzipien des ärztlichen Handelns zusammen und ist in vielen Ländern - so auch in Deutschland - Teil der ärztlichen Berufsordnung. Im Oktober 2017 hat der Weltärztebund eine überarbeitete Fassung des zeitgemäßen hippokratischen Eids für Ärzte veröffentlicht. Neu ist das Versprechen, auch die eigene Gesundheit zu pflegen: „Ich werde auf meine eigene Gesundheit, mein Wohlergehen und meine Fähigkeiten achten, um eine Behandlung auf höchstem Niveau leisten zu können.“

Gesundheit und Wohlergehen von Ärztinnen und Ärzten sind in den letzten Jahren zunehmend in den Fokus von Politik und Öffentlichkeit gerückt; der 122. Deutsche Ärztetag 2019 in Münster hat die Gesundheit und die Gesund-

Klaus Reinhardt, Bundesärztekammer

Ә OpenAccess. () 2021 Klaus Reinhardt, publiziert von De Gruyter. (cc))BY-NC-SA Dieses Werk ist lizenziert unter einer Creative Commons Namensnennung - Nicht kommerziell - Weitergabe unter gleichen Bedingungen 4.0 International Lizenz. https://doi.org/10.1515/9783110713336-023 
erhaltung von Ärztinnen und Ärzten in einem eigenen Tagesordnungspunkt und im Kontext der Arbeitsbedingungen im deutschen Gesundheitswesen hervorgehoben. Dieser Beitrag soll die gesundheitlichen Gefährdungen und Folgen aufzeigen, die sich im ärztlichen Berufsalltag aufgrund der aktuellen Arbeitsbedingungen ergeben. Er soll Lösungsansätze aufzeigen, die die Arztgesundheit und damit auch die Patientenversorgung mittel- und langfristig verbessern können.

\section{Gesundheitsrisiken für Ärztinnen und Ärzte in der medizinischen Versorgung}

Ärztinnen und Ärzte gehen bei der Ausübung ihres Berufes gesundheitliche Risiken ein, die sich zu einem Teil zwar minimieren, nie aber ganz vermeiden lassen. Gefahren für die Gesundheit des medizinischen Personals ergeben sich aus dem Umgang mit infektiösen Materialien, Gefahrenstoffen, Strahlung und Instrumenten sowie durch Schäden aufgrund einseitiger Belastungen und Fehlhaltungen bei Untersuchungen und Eingriffen. Auch psychische Belastungen aufgrund der hohen Verantwortung für Leben und Wohlergehen der ihnen anvertrauten Menschen sowie des Erlebens von Leid und Sterben gehören seit jeher zum Arztberuf, wenngleich ihre Auswirkungen lange Zeit wenig Beachtung fanden.

Aktuelles Beispiel für die Risiken einer Tätigkeit im medizinischen Bereich ist die SARS-CoV-2-Pandemie, bei der sich, vor allem durch eine vollkommen unzureichende Versorgung mit Schutzausrüstungen, weltweit Ärzte und medizinisches Personal bei der Versorgung von Patienten infiziert haben. Exemplarisch für diese Gefährdung steht der Tod des 34-jährigen Arztes Li Wenliang aus Wuhan, der als Erster in sozialen Netzwerken vor dem Coronavirus warnte, selbst infiziert wurde und an COVID-19 verstarb. In Deutschland war laut Lagebericht des Robert KochInstituts bis Mitte Juni 2020 bei 14.443 Infizierten (darunter 23 Todesfällen) bekannt, dass sie in einer medizinischen Einrichtung gemäß § 23 IfSG (z. B. Krankenhäuser, ärztliche Praxen, Dialyseeinrichtungen und Rettungsdienste) tätig waren (Robert Koch-Institut 2020). Die tatsächliche Zahl dürfte aufgrund unvollständiger Datenerhebung bei der Meldung noch deutlich höher liegen. Die WHO nennt in diesem Zusammenhang weitere Risiken für das in die Versorgung von COVID-19-Patienten eingebundene medizinische Personal: die hohe zeitliche Arbeitsbelastung, psychischer Stress, Erschöpfung, Burnout sowie physische und psychische Gewalterlebnisse (World Health Organization 2020).

Auch wenn die Corona-Pandemie ein extremes Versorgungsszenario darstellt, sind die von der WHO genannten Faktoren auch in der Versorgung unter „Nor- 
malbedingungen“ längst Realität. Gesundheitssysteme drohen unter der Last der Pandemie zusammenzubrechen, unter Alltagsbedingungen funktionieren sie teilweise nur, weil das medizinische Personal trotz widriger Rahmenbedingungen die Versorgung aufrechterhält und Raubbau an der eigenen Gesundheit betreibt.

\section{Arbeitsbelastung in der ärztlichen Tätigkeit}

Ärztinnen und Ärzte haben eine hohe zeitliche Arbeitsbelastung. Dies betrifft sowohl die Anzahl von Überstunden, die tatsächliche Beanspruchung während der Bereitschaftsdienste, die Möglichkeiten, Pausenzeiten einhalten zu können, und die Arbeit an Wochenenden, in der Freizeit und im Urlaub. Insgesamt liegt die Arbeitszeit der Ärzte zum Teil ganz erheblich über den gesetzlichen Vorgaben, mit negativen Folgen für die Vereinbarkeit von Familie und Beruf und die Work-LifeBalance (Hartmannbund 2019; Hussenöder et al. 2020; Kassenärztliche Bundesvereinigung \& Verband der niedergelassenen Ärzte Deutschlands e.V. 2018; Marburger Bund 2020; Raspe et al. 2019).

Ein wichtiger Grund für die hohe zeitliche Beanspruchung ist der Ärztemangel beziehungsweise die unzureichende Personalausstattung des Gesundheitswesens, nicht nur im ärztlichen Bereich, sondern auch im Bereich der Pflege. Deutsche Ärztetage haben wiederholt für eine angemessene ärztliche Personalausstattung im stationären Bereich plädiert. Zahlreiche von Abgeordneten des Ärztetags eingebrachte Beschlussanträge haben in eindringlichen Appellen Personalvorgaben für Ärzte gefordert, die eine patienten- und aufgabengerechte Versorgung ermöglichen. In der Hartmannbund-Assistenzarztumfrage 2018/2019 wurde die Personaldecke in den Abteilungen von einem Drittel der Befragten als mangelhaft bis ungenügend eingeschätzt. Die Hälfte der Befragten gab an, dass ein Puffer für Personalausfälle fehle. Dies hat Auswirkungen auf die Zeit, die verbleibt, um sich direkt den Patienten widmen zu können, und führt zu einer deutlichen Verdichtung der Arbeit (Hartmannbund 2019).

Im ambulanten Bereich bedingen demographischer Wandel, vermehrte Tätigkeiten in Anstellung und Teilzeit sowie der zunehmende Ärztemangel insbesondere in strukturschwachen und ländlichen Gebieten überfüllte Arztpraxen und eine hohe Arbeitsbelastung.

Die zunehmende Bürokratisierung der Medizin als Folge umfänglicher sozialrechtlicher und wirtschaftlicher Vorgaben ist ein weiteres Problem im Berufsalltag von Ärzten (Kassenärztliche Bundesvereinigung \& Verband der niedergelassenen Ärzte Deutschlands e.V. 2018). Der Bürokratieindex für die vertragsärztliche Versorgung von 2019 geht von etwa 60 Arbeitstagen aus, die pro Jahr und Praxis für die Erfüllung von Informationspflichten aufgewendet werden 
müssen (Kassenärztliche Bundesvereinigung 2019). In der Hartmannbund-Umfrage gaben 80 Prozent der Befragten an, bis zu 50 Prozent ihrer Arbeitszeit mit Tätigkeiten wie Dokumentation, Telefonaten, Befund-Recherchen, Arztbriefen, Rehabilitations-Anträgen und der Organisation von Terminen zu verbringen (Hartmannbund 2019). Gespräche mit Patienten, Angehörigen und Kollegen müssen häufig auf ein Minimum reduziert werden, oft fehlt sogar die Zeit für Essen und Trinken.

Daneben fühlen sich vor allen Dingen junge Ärzte oftmals mit ihren Sorgen, den anspruchsvollen Tätigkeiten und der hohen Verantwortung für ihre Patienten allein gelassen. Sie beklagen teilweise eine mangelnde Unterstützung durch erfahrene Ärzte (Hartmannbund 2019), die aber häufig ebenfalls der unzureichenden Personalausstattung geschuldet ist.

\section{Gewalt gegen Ärztinnen und Ärzte}

Neben der hohen Arbeitsbelastung stellt Gewalt durch Patienten bzw. Angehörige eine unmittelbare Bedrohung für die physische und psychische Gesundheit von Ärzten und ihren Mitarbeitern dar. Berichte zu Gewalt gegen medizinisches Personal haben in den letzten Jahren deutlich zugenommen, insbesondere Notärzte und Rettungssanitäter wurden wiederholt angegriffen. Betroffen sind außerdem Arbeitsbereiche mit direktem Patientenzugang wie Notaufnahmen, Ambulanzen und Kreißsäle (Lindner et al. 2015). Auch in Praxen niedergelassener Ärzte zeigen sich Gewalterlebnisse erschreckenden Ausmaßes (Kassenärztliche Bundesvereinigung \& Verband der niedergelassenen Ärzte Deutschlands e.V. 2018; Vorderwülbecke et al. 2015).

$\mathrm{Zu}$ Gewaltereignissen kommt es häufig in Situationen, in denen Ärzte ohnehin an die Grenzen ihrer körperlichen und mentalen Belastbarkeit gelangen. Überfüllte Notaufnahmen und Wartezimmer, Patientinnen und Patienten mit Erwartungen, die nicht erfüllt werden können, erzeugen Anspannung und Stress. Gewalt kann in diesen Situationen zusätzlich ein Gefühl von Unzulänglichkeit und Überforderung auslösen.

\section{Kommerzialisierung des Gesundheitswesens}

Die Ursachen für die hohe Arbeitsbelastung von Ärztinnen und Ärzten lassen sich auch auf das Problem der zunehmenden Kommerzialisierung in der Medizin zurückführen, die zu der vollkommen unzureichenden Personalausstattung beiträgt und so letztendlich die Arbeitsbelastung erhöht. Wirtschaftliches Verhalten ist, 
gerade in einem Solidarsystem, eine wichtige Voraussetzung für ein nachhaltiges und funktionierendes Gesundheitssystem. Dennoch dürfen wirtschaftliche Prämissen nicht über die Bedarfe und Bedürfnisse von Patientinnen und Patienten und das Wohl der Mitarbeiter gestellt werden. Krankenhäuser sind einem zunehmenden wirtschaftlichem Leistungsdruck ausgesetzt, viele Krankenhäuser befinden sich in einer schlechten wirtschaftlichen Verfassung mit steigender Insolvenzwahrscheinlichkeit (Augurzky et al. 2020) und müssen zunehmend profitorientiert agieren. Da die Personalkosten im Gesundheitsbereich mit rund 60 Prozent den größten Kostenfaktor darstellen (Destatis 2018), werden Einsparungen vor allen Dingen in diesem Bereich vorgenommen. Inzwischen wurden, um hier Abhilfe zu schaffen, die Pflege-Personalkosten aus dem Finanzierungssystem ausgegliedert. Das ist gut und richtig, zu konstatieren ist aber auch, dass sich seither der Druck auf das ärztliche Personalbudget weiter verschärft. Gleichzeitig hat sich die Arbeit in den Krankenhäusern in den vergangenen Jahren auf Grund eines immer höheren Patientendurchsatzes und immer kürzerer Verweildauern deutlich verdichtet. Patienten müssen in kürzester Zeit alle diagnostischen und therapeutischen Prozesse durchlaufen, um Kosten zu sparen und Sanktionen zu vermeiden, die bei einer Überschreitung festgelegter Verweildauern fällig werden. Grundleistungen wie ärztliche Gespräche und Beratungen sowie der interdisziplinäre Austausch in Klinik und Praxis werden dagegen nicht adäquat finanziert. Die Folge ist, dass finanzielle Mittel, insbesondere aus dem Personalbereich, für dringende Investitionen zweckentfremdet werden, was den Arbeitsdruck weiter erhöht. Die Ärzteschaft fordert deshalb seit langem, dass die Vergütungssystematik der Krankenhäuser nicht länger ausschließlich auf wirtschaftliche Effizienz eines Krankenhausbetriebes hin ausgerichtet wird, sondern sich die Vergütung vielmehr nach der tatsächlichen Behandlungsbedürftigkeit des einzelnen Patienten richtet und die Angemessenheit der Behandlung honoriert. Zudem ist dringend erforderlich, dass die Bundesländer in vollem Umfang ihren Investitionsverpflichtungen für den stationären Bereich nachkommen und den Investitionsstau von rund 30 Milliarden Euro auflösen.

\section{Auswirkungen der Arbeitsbelastung auf die Arztgesundheit}

Glücklicherweise gelingt es vielen Ärztinnen und Ärzten immer noch, gut mit Stressoren in ihrem Arbeitsalltag umzugehen. Niedergelassene Ärzte konstatieren insgesamt eine hohe Berufszufriedenheit, die meisten Ärzte empfinden ihre Tätigkeit als nützlich und sinnvoll und würden den Beruf erneut ergreifen 
(Kassenärztliche Bundesvereinigung \& Verband der niedergelassenen Ärzte Deutschlands e.V. 2018). Auch in einer Umfrage unter sächsischen Ärzten wurde insbesondere das hohe Sinnerleben hervorgehoben (Hussenöder et al. 2020).

Das Erleben, eigenen Qualitätsansprüchen an die Patientenversorgung aus Zeitmangel und Überforderung nicht genügen zu können, stellt für Ärzte allerdings eine immense seelische Belastung dar. Bereits 2018 sprach der damalige Präsident des Weltärztebundes, Prof. Dr. Leonid Eidelman, von einer weltweiten „pandemic of physician burnout“. Er warnte, dass fast die Hälfte der weltweit zehn Millionen Ärzte Symptome eines Burnouts wie emotionale Erschöpfung, zwischenmenschliche Distanzierung und vermindertes Zutrauen in die eigene Leistungsfähigkeit zeigen würden (World Medical Association 2018).

Viele Ärzte berichten schon in jungen Jahren von einer Erschöpfungssymptomatik und zwischenmenschlicher Entfremdung. Dies kann in eine Erschöpfungsdepression münden, aber auch ungesunde Verhaltensweisen zur Folge haben, wie z.B. einen erhöhten Suchtmittelgebrauch (Raspe et al. 2019).

Wissenschaftliche Studien zeigen eine erhöhte Krankheitslast von Ärzten insbesondere für Depressionen, Angst und Burnout auf, die über der der Normalbevölkerung liegt (Angerer et al. 2007; Gottschling 2019; Mata et al. 2015).

Arbeitsverdichtung, Schlafmangel, Daueranspannung, aber auch der Umgang mit menschlichem Leid, hohe Verantwortung und das Erleben von Hilflosigkeit im Angesicht unheilbarer Erkrankungen können bei prädisponierten Ärzten auch zu Suchterkrankungen führen.

\section{Folgen der Arbeitsbelastung für die Patientenversorgung}

Die hohe Arbeitsbelastung und auch die damit verbundenen gesundheitlichen Folgen für Ärztinnen und Ärzte können die Versorgungsqualität und die Patientensicherheit beinträchtigen. So ist ärztliches Burnout mit einem erhöhten Risiko reduzierter Patientensicherheit und Patientenzufriedenheit sowie reduzierter Versorgungsqualität assoziiert, die sich an Kriterien wie vermehrten Behandlungskomplikationen, Medikationsfehlern und fehlender Berücksichtigung wissenschaftlicher Leitlinien sowie fehlender Empathie festmachen lassen (Fahrenkopf et al. 2008; Klein et al. 2011; Loerbroks et al. 2017; Panagioti et al. 2018).

Auch ein häufiges Erleben von verbalen Aggressionen geht mit einer subjektiv geringeren Versorgungsqualität einher (Raspe et al. 2019). Stress und Überlastung können sich auch auf das Verhalten von Ärzten auswirken. Von Ärzten genannt 
werden Gereiztheit, Unfreundlichkeit, reduziertes Engagement, Aufmerksamkeitsdefizite und Fehleranfälligkeit (Gottschling 2019).

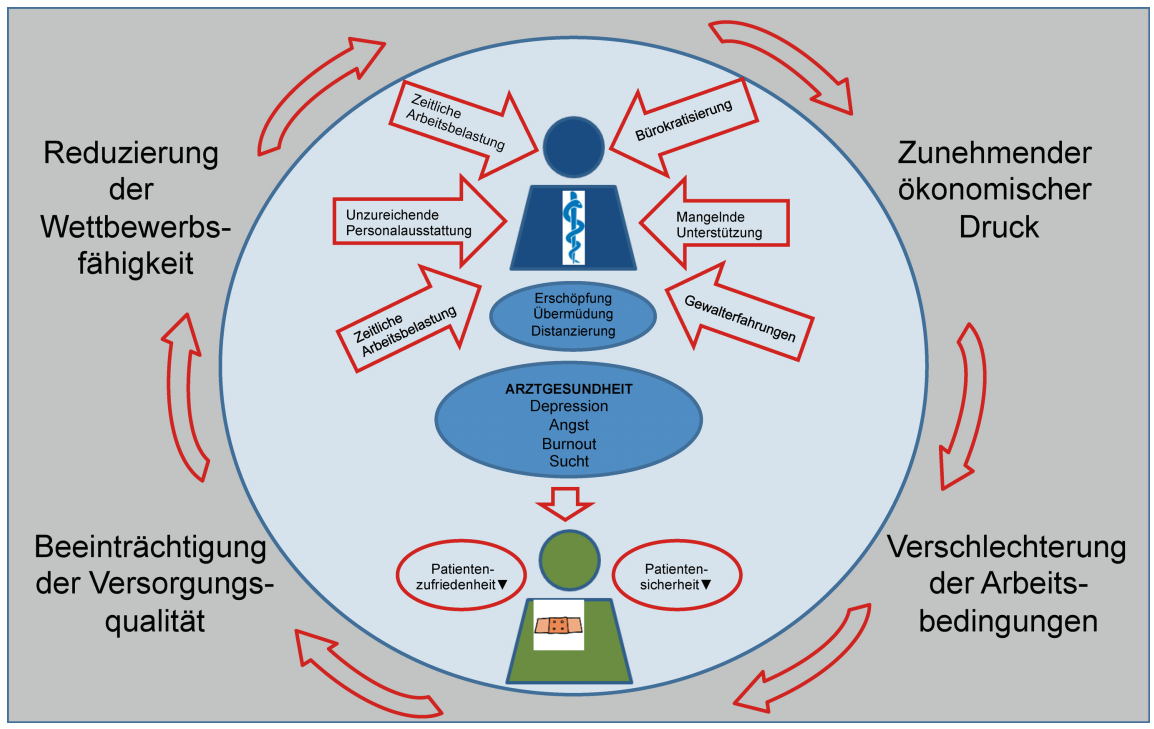

Abb. 1: Arztgesundheit und Versorgungsqualität im Circulus vitiosus der Kommerzialisierung des Gesundheitswesens (Eigene Abbildung)

\section{Lösungsansätze zur Förderung der Arztgesundheit}

Als wesentliche Voraussetzungen für gesundheitsförderliche Arbeitsbedingungen in den Krankenhäusern und Arztpraxen sieht die Ärzteschaft gesetzliche und ökonomische Rahmenbedingungen, die der Kommerzialisierung des Gesundheitswesens entgegenwirken, die Personalausstattung in den Kliniken verbessern, die Bürokratisierung abbauen und den Mangel an Arztstunden beheben.

Der 122. Deutsche Ärztetag 2019 in Münster hat die „Arztgesundheit“ zu einem Schwerpunktthema gewählt und zahlreiche Beschlüsse gefasst, die Lösungswege für Erhalt und Förderung insbesondere auch der seelischen Gesundheit von Ärzten aufzeigen.

Ärzte mit manifesten Berufskrankheiten, mit Depression und Burnout oder Suchterkrankungen müssen unterstützt und behandelt werden. Die Ärztekammern bieten zum Beispiel vertrauliche Hilfs- oder Interventionsprogramme für suchtkranke Ärzte an. Wichtig sind außerdem Angebote der Primär-, Sekundär- 
und Tertiärprävention gegen körperliche und verbale Gewalt am Arbeitsplatz. Die Ärztekammern bieten hierzu eine Vielzahl von Beratungs- und Fortbildungsangeboten an. Für Beschäftigte mit posttraumatischem Syndrom (PTS) gibt es Unterstützungsangebote der Berufsgenossenschaft für Gesundheitsdienst und Wohlfahrtspflege (BGW), aber auch Arbeitgeber sollten niedrigschwellige Hilfsangebote bereitstellen, die helfen, dass traumatische dienstliche Erlebnisse adäquat verarbeitet werden.

Daneben müssen auf der individuellen und der betrieblichen Ebene Maßnahmen entwickelt und umgesetzt werden, die die Resilienz von Ärzten stärken, frühzeitig seelische Belastungen erkennen und entsprechende Interventionen ermöglichen. Dazu gehören auch lebensphasengerechte Präventionsmodelle und Unterstützungsangebote (z. B. flexible Arbeitszeitmodelle), damit Beruf, Familie, Freizeit und Pflege von Angehörigen besser miteinander vereinbar werden. Angebote der gesetzlichen Unfallversicherungsträger und des staatlichen Arbeitsschutzes sollten vermehrt nachgefragt und das betriebliche Gesundheitsmanagement gestärkt werden.

Für eine hohe Arbeitszufriedenheit und Resilienz spielt auch die intra- und interprofessionelle Zusammenarbeit eine wichtige Rolle. Durch mehr Kooperation und Vernetzung zwischen Hausärzten, Fachärzten, Krankenhausärzten und den Gesundheitsfachberufen lässt sich die Versorgung patientenzentriert organisieren und dem Effizienz- und Wirtschaftlichkeitsdruck eher begegnen. Die Förderung von Organisations- und Personalentwicklung, der Abbau starrer Hierarchien und ein an der Gesundheit der Mitarbeiterinnen und Mitarbeiter orientierter, wertschätzender und kooperativ ausgerichteter Führungsstil sind weitere Maßnahmen, die zu einem gesunden Arbeitsumfeld beitragen. Wichtig ist zudem ein umfassendes Angebot der Aus-, Fort- und Weiterbildung zu Themen wie Resilienz, Stressbewältigung, ärztliche Führung und Teamentwicklung, um bereits frühzeitig entsprechende Kompetenzen zu stärken.

Eine große Bedeutung kommt der ärztlichen Führung im Krankenhaus zu. Medizinethiker plädieren dafür, dass ethische Vorgaben integraler Bestandteil des Krankenhaus-Managements werden. Dafür muss eine klare Definition der normativen Vorgaben, z. B. in Form eines Leitbildes, erarbeitet und durch wiederholte Mitarbeiterbefragungen in der Routine der Krankenhausversorgung systematisch überprüft werden. Mehr Ethik im Krankenhaus werde sich letztendlich durch eine Steigerung der wirtschaftlichen Leistungsfähigkeit auszahlen (Marckmann \& Maschmann 2017).

Die Arbeitgeber im Gesundheitswesen müssen zudem ihrer Verpflichtung für die Schaffung gesundheitsgerechter Arbeitsbedingungen deutlich stärker als bisher nachkommen. Dies bedeutet konkret, Arbeitsschutzregelungen einschließlich des Arbeitszeitgesetzes (ArbZG) konsequent einzuhalten und Perso- 


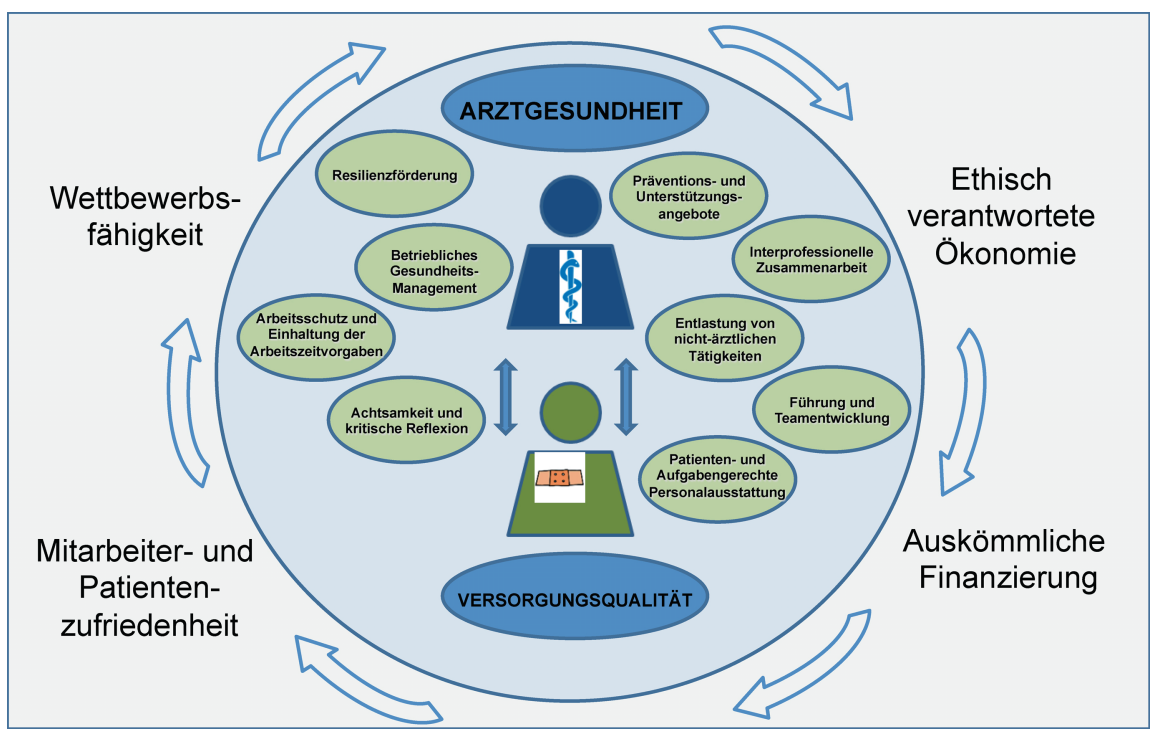

Abb. 2: Voraussetzungen für die Steigerung und den Erhalt von Arztgesundheit und Versorgungsqualität (Eigene Abbildung)

nalschlüssel auf arbeitswissenschaftlicher Grundlage auszugestalten, so dass eine patienten- und aufgabengerechte Versorgung $\mathrm{zu}$ jeder Zeit möglich ist.

Ärzte müssen zudem stärker von Verwaltungstätigkeiten und anderen nichtärztlichen Tätigkeiten entlastet werden, um mehr Zeit für die direkte Patientenversorgung zu haben. Hier wird auch die digitale Transformation eine große Rolle spielen, sofern es gelingt, die digitalen Anwendungen des Gesundheitswesens so auszugestalten, dass Patienten und Personal davon profitieren und Vertrauen in die neuen Strukturen und Abläufe entwickeln können.

Daneben sind auch die Ärzte selbst gefragt, sich kritisch mit den Arbeitsbedingungen auseinanderzusetzen, Verstöße gegen geltende Arbeitszeitregelungen nicht länger hinzunehmen, sich gegen Missstände, die die Qualität der Patientenversorgung beeinträchtigen, zur Wehr zu setzen und sich auch berufspolitisch zu engagieren. Ärzte müssen auf ihre eigene Gesundheit achten und sich dagegen verwahren, dass ökonomische Zwänge und unhaltbare Arbeitsbedingungen ihnen die Freude und Begeisterung am Beruf und am Einsatz für ihre Patienten nehmen.

Unser Ziel muss ein humanes Gesundheitswesen sein, das nicht „durchökonomisiert“ ist wie ein Industriebetrieb. Patienten haben einen berechtigten Anspruch darauf, angemessen behandelt zu werden - nicht nur technisch, sondern vor allem auch menschlich. 


\section{Literatur}

Angerer, Peter/Petru, Raluca Nowak, Dennis et al. (2007): „Arbeitsbedingungen und Depression bei Ärzten“. In: DMW - Deutsche Medizinische Wochenschrift 133(01/02), S. $26-29$.

Augurzky, Boris/Krolop, Sebastian/Pilny, Adam et al. (2020): Krankenhaus Rating Report 2020: Ende einer Ära. Aufbruch ins neue Jahrzehnt. Heidelberg: Medhochzwei.

Destatis (2018): Gesundheit: Kostennachweis der Krankenhäuser 2017 Fachserie 12 Reihe 6. 3. Wiesbaden: Statistisches Bundesamt, https:/www.destatis.de/DE/Themen/GesellschaftUmwelt/Gesundheit/Krankenhaeuser/Publikationen/Downloads-Krankenhaeuser/ kostennachweis-krankenhaeuser-2120630177004.pdf?_blob=publicationFile, besucht am 5.8.2020.

Fahrenkopf, Amy M./Sectish, Theodore C./Barger, Laura K. et al. (2008): „Rates of Medication Errors among Depressed and Burnt Out Residents: Prospective Cohort Study“. In: Bmj 336(7642), S. $488-491$.

Gottschling, Claudia (2019): „Medscape-Report: Burnout und Depression sind weit verbreitet. Warum der Arztberuf krankmachen kann und was davor schützt“. https://deutsch.staging. medscape.com/diashow/49000679? faf=1, besucht am 5.8.2020.

Hartmannbund (2019): Die große HB-Assistenzarztumfrage 2018/2019: Zwischen Arbeitszeit, Fehlermanagement und Digitalisierung. Wie gut können Sie gute Ärzte werden?, https:// www.hartmannbund.de/fileadmin/user_upload/Downloads/Umfragen/HB-

Assistenzarztumfrage-2018-2019.pdf, besucht am 5.8.2020.

Hussenöder, Felix S./Conrad, Ines/Riedel-Heller, Steffi (2020): Berufliche Belastung, Gesundheitszustand und Berufszufriedenheit sächsischer Ärztinnen und Ärzte 2019 - eine Folgebefragung. Dresden: Sächsische Landesärztekammer, https://www.slaek.de/media/ dokumente/05slaek/publikation/studiebroschur/Berufliche_Belastung_ Gesundheitszustand_Studie.pdf, besucht am 5.8.2020.

Kassenärztliche Bundesvereinigung (2019): „Bürokratieindex (BIX)“. https://www.kbv.de/html/ bix.php, besucht am 5.8.2020.

Kassenärztliche Bundesvereinigung/Verband der niedergelassenen Ärzte Deutschlands e.V. (2018): Tabellenband Ärztemonitor 2018: Ergebnisse für Haus- und Fachärzte. Bonn: infas Institut für angewandte Sozialwissenschaft GmbH, https://www.kbv.de/media/sp/infas_ TabBand_Aerztemonitor2018_Aerzte_20180615.pdf, besucht am 5.8.2020.

Klein, Jens/Frie, Kirstin Grosse/Blum, Karl et al. (2011): „Psychosocial Stress at Work and Perceived Quality of Care among Clinicians in Surgery“. In: BMC health services research 11(1), Article number: 109.

Lindner, Tobias/Joachim, R./Bieberstein, Stefanie. et al. (2015): „Aggressives und herausforderndes Verhalten gegenüber dem Klinikpersonal“. In: Notfall + Rettungsmedizin 18(3), S. $195-200$.

Loerbroks, Adrian/Glaser, Jack/Vu-Eickmann, Patricia et al. (2017): „Physician Burnout, Work Engagement and the Quality of Patient Care“. In: Occupational Medicine 67(5), S. 356-362.

Marburger Bund (2020): „MB-Monitor 2019: Überlastung führt zu gesundheitlichen Beeinträchtigungen“. https://www.marburger-bund.de/mb-monitor-2019, besucht am 5.8.2020. 
Marckmann, Georg/Maschmann, Jens (2017): „Ethische Mangelverwaltung“. In: Deutsches Ärzteblatt 114(44), S. A 2028-2032.

Mata, Douglas A./Ramos, Marco A./Bansal, Narinder et al. (2015): „Prevalence of Depression and Depressive Symptoms Among Resident Physicians“. In: Jama 314(22), S. 2373.

Panagioti, Maria/Geraghty, Keith/Johnson, Judith et al. (2018): „Association Between Physician Burnout and Patient Safety, Professionalism, and Patient Satisfaction“. In: JAMA Internal Medicine 178(10), S. 1317.

Raspe, Matthias/Koch, Peter/Zilezinski, Max et al. (2019): „Arbeitsbedingungen und Gesundheitszustand junger Ärzte und professionell Pflegender in deutschen Krankenhäusern“. In: Bundesgesundheitsblatt - Gesundheitsforschung Gesundheitsschutz 63(1), S. 113-121.

Robert Koch-Institut (2020): „Täglicher Lagebericht des RKI zur Coronavirus-Krankheit-2019 (COVID-19). 04.08.2020 - Aktualisierter Stand für Deutschland“. https://www.rki.de/DE/ Content/InfAZ/N/Neuartiges_Coronavirus/Situationsberichte/2020-08-04-de.pdf?_blob= publicationFile, besucht am 5.8.2020.

Vorderwülbecke, Florian/Feistle, Maximilian/Mehring, Michael et al. (2015): „Aggression and Violence Against Primary Care Physicians“. In: Deutsches Aerzteblatt Online. https://www. aerzteblatt.de/archiv/168409/Aggression-und-Gewalt-gegen-Allgemeinmediziner-undpraktische-Aerzte, besucht am 5.8.2020

Weltärztebund (2017): „Deklaration von Genf. Deutsche Fassung 2017“. https://www. bundesaerztekammer.de/fileadmin/user_upload/downloads/pdf-Ordner/International/ Deklaration_von_Genf_DE_2017.pdf, besucht am 5.8.2020.

World Health Organization (2020): „Coronavirus Disease (COVID-19) Outbreak: Rights, Roles and Responsibilities of Health Workers, Including Key Considerations for Occupational Safety and Health. Interim Guidance“. https://www.who.int/publications/i/item/ coronavirus-disease-(covid-19)-outbreak-rights-roles-and-responsibilities-of-healthworkers-including-key-considerations-for-occupational-safety-and-health, besucht am 5.8.2020.

World Medical Association (2018): „WMA Leader Warns of Global Physician Burnout“. https:// www.wma.net/news-post/wma-leader-warns-of-global-physician-burnout/, besucht am 5.8.2020. 
